\title{
Evaluación de la pérdida de suelo, asociada al proceso de expansión urbana y reconversión productiva. Caso: comunas de Los Andes, Quillota y Concón, valle del Aconcagua ${ }^{1}$
}

\author{
Carmen Paz Castro Correa ${ }^{2}$ y Cristóbal Aliaga ${ }^{3}$
}

\begin{abstract}
RESUMEN
La evaluación de la vulnerabilidad a la degradación de suelos de las comunas de Los Andes, Quillota y Concón, representativas de diferentes ambientes morfoclimáticos del valle del Aconcagua, se realizó a través de la identificación de Unidades Homogéneas de Respuesta a la Erosión (ERU). Posteriormente, se aplicó la Ecuación Universal de Pérdida de Suelo (USLE), en microcuencas representativas, simulando dos distintos escenarios representativos de diferentes prácticas de conservación de suelos. Por otra parte, se aplicaron indicadores de calidad de suelo en el fondo de valle, para cuantificar la degradación producida en la última década.

Los resultados obtenidos muestran una degradación de los suelos agrícolas del valle, producto tanto de factores endógenos como exógenos, entre los que la topografía y la cobertura vegetal resultan relevantes, sumados a los impactos de la dinámica socioeconómica. En los suelos agrícolas del fondo de valle muestran la mayor vulnerabilidad del área de estudio, dada la magnitud de las transformaciones territoriales que han presentado en las últimas décadas.
\end{abstract}

Palabras clave: Erosión, Unidades de Respuesta a Erosión (ERU), USLE.

\begin{abstract}
The assessment of soil degradation in Los Andes, Quillota and Concon municipalities, which represents the morphoclimatic environments of Aconcagua Valley, was identified through the Erosion Respones Units (ERUs). Subsequently, the soil loss was quantified through the Universal Soil Loss Equation (USLE) in microbasins, and timely simulations were made for two different scenarios, representing the implementation of soil conservation practices. On the other hand, quality indicators were used to quantify the degradation produced in the last decade on the bottom part of the valley.

Results show a clear degradation of agricultural valley soils as a result of both endogenous and exogenous factors. Among them, the topography and vegetation coverage were found to be relevant, in addition to the impact of socio-economic dynamics. Agricultural soils from the bottom part of the valley show the highest vulnerability of the study, due to intense territorial changes in the last decades.
\end{abstract}

Key words: Soil Degradation (Erosion), Erosion Response Units (ERU), USLE.

1 Proyecto FONDECYT No 1071098. Artículo recibido el 9 de marzo de 2009 y aceptado el 17 de abril de 2009.

2 Departamento de Geografía, Universidad de Chile (Chile). E-mail: cpcastro@uchile.cl
3 Departamento de Geografía, Universidad de Santiago de Chile (Chile). E-mail: cristobal.aliaga@usach.cl 
En las últimas dos décadas, Chile ha experimentado un importante crecimiento económico que se ha visto reflejado en un importante desarrollo y modernización agrícola de sus cuencas y en una expansión de sus ciudades interiores y costeras, entre otros. Sin embargo, los beneficios que este proceso ha traído consigo, en especial en aquellos territorios de clara vocación exportadora, podrían estar generando significativos impactos ambientales.

La magnitud de estos impactos está relacionada, en gran medida, con la vulnerabilidad a la degradación del sistema natural en que se han desarrollado los cambios en el uso y manejo del suelo.

La cuenca del río Aconcagua, con excelentes condiciones climáticas y edáficas, se ha incorporado desde hace tres décadas al proceso de modernización agrícola, sufriendo un acelerado cambio de uso del suelo. La expansión de sus fronteras agrícolas y urbanas ha generado, además, conurbaciones de importancia como las observadas entre Quillota y La Cruz (Castro y Ortiz, 2005).

En este escenario se evaluó la degradación potencial y diferencial (Fournier, 1975), que puede estar sufriendo la cuenca, según susceptibilidad morfoedafológica, posición morfoclimática y grado de intervención correspondiente, lo que permite una evaluación presente y futura del paisaje en términos de su capacidad de sostener las actividades productivas y de asentamientos humanos, además de establecer tendencias e identificar y corregir impactos (Aliaga y Leyton, 2007).

Enmarcado en una modelación del paisaje y en una estimación de la erosión, el modelamiento de las ERU permite dividir un área en regiones con similar grado y clase de susceptibilidad a la erosión.

\section{Metodología}

Los modelos utilizados son básicamente cualitativos y cuantitativos. El primero, en base a las unidades de respuesta a la erosión, considera las condiciones del terreno (geomorfología, geología, pendientes) y las condiciones climáticas predominantes en el área de estudio, así como las características de eventos excepcionales de precipitaciones intensas. Para el caso de la pérdida de suelo, se analizó su erosionabilidad, considerando la cobertura vegetacional, la pendiente y las características morfológicas y fisicoquímicas de este.

\section{Selección de variables físico-naturales}

Se evaluaron en primer lugar las unidades de respuesta a la erosión (modelo de evaluación cualitativo de los procesos de erosión) para las comunas de Concón, Quillota y Los Andes, de acuerdo a lo expuesto por Märker et al. (2001), generando como resultado una cartografía de susceptibilidad a la erosión por comunas.

Por otra parte, de manera de complementar la información anterior y de cuantificar la pérdida de suelo dentro de cada comuna, se seleccionaron microcuencas adecuadas para la aplicación de la Ecuación Universal de Pérdida de Suelo (USLE) y se aplicaron indicadores de calidad de suelo en el fondo de valle.

\section{Unidades de Respuesta a la Erosión (ERU)}

Las ERU (Erosion Response Units), en el contexto de los modelos de erosión cualitativos, se definen como unidades espaciales heterogéneamente localizadas, pero homogéneas en cuanto al funcionamiento de sus procesos físico-naturales y con un determinado nivel de susceptibilidad a la erosión, controlado por sus propiedades fisiográficas y el manejo de su ambiente natural y humano (Märker et al., 2001). Estas entidades espaciales se generan a partir de la sobreposición ponderada de las distintas variables del medio físico-natural y la identificación de las denominadas Unidades de Referencia a la Erosión (ERefU), que describen los procesos erosivos actuales y las formas del paisaje existente.

La superposición consideró el análisis de fotografías áreas, ortofotos, visitas a terreno y procesamiento SIG (Figura $N^{\circ} 1$ ). La identificación y localización de las formas erosivas existentes (ERefU) se realizó clasificando los tipos de erosión según profundidad y 
espacio entre regueras y/o cárcavas (adaptado de la metodología de Van Zuidam, 1985) y las intensidades de degradación estimada en función de la densidad de la cobertura vegetal.

De esta manera, todas las variables utilizadas para la definición de las Unidades de Respuesta a Erosión (ERU) en la cuenca del Aconcagua, fueron evaluadas en forma independiente según su aporte en el proceso de erosión, estableciendo niveles de erodabilidad o erosividad, según correspondiere. Con el fin de espacializar dichos análisis, cada una de las variables involucradas fue trabajada como una cobertura de información formato grilla en el Sistema de Información Geográfica ArcGIS 9.1 (Figura № 2).

Figura $\mathrm{N}^{\circ} 1$

Sobreposición de los parámetros utilizados para la determinación de ERU

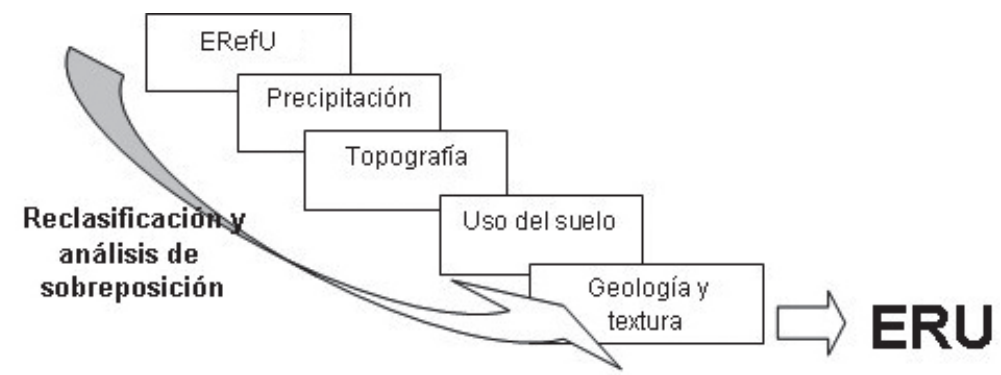

Fuente: Adaptado de Märker et al., 2001.

Figura $\mathrm{N}^{\circ} 2$

Diagrama de los procesos del ecosistema entendido como

"Unión de intercambio suelo-vegetación-atmósfera"

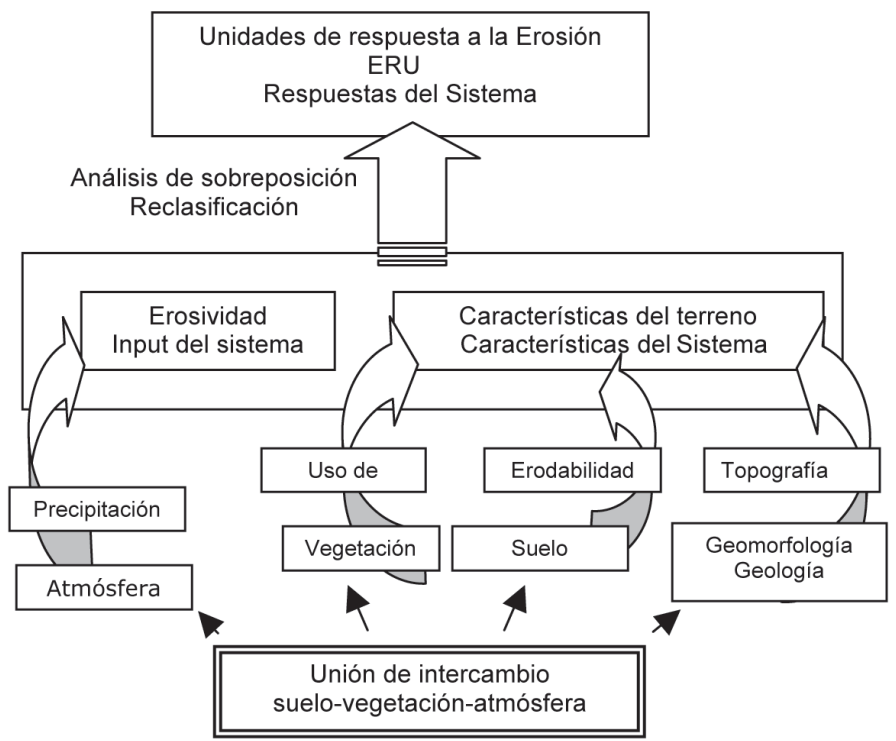

Fuente: Adaptado de Märker et al., 2001. 


\section{Ecuación Universal de Pérdida de Suelo (USLE)}

Dentro de los modelos empíricos que destacan por su amplia utilización y simplicidad de aplicación, el más utilizado ha sido el de la Ecuación Universal de Pérdida de Suelo (USLE), especialmente adaptado para evaluar las pérdidas de suelo por erosión laminar y en surcos. En Chile, diversos investigadores, como Honorato y Cruz (1999) entre otros, han aplicado el modelo USLE, considerando variados medios climáticos y edáficos, así como tipos de manejo de suelo, obteniendo una buena correspondencia entre las estimaciones del modelo y los resultados de las parcelas de erosión (Honorato et al., 2001).

En este estudio, la ecuación se aplicó a microcuencas seleccionadas como representativas dentro de las comunas de Concón, Quillota y Los Andes. Con la definición y cálculo de cada uno de los parámetros del modelo y la utilización del SIG ArcGIS 9.1 (Spatial analyst), se obtuvo un Valor de Pérdida de Suelo A (ton/ha*año) para cada una de las microcuencas en estudio.

\section{Resultados}

A través del modelamiento de las ERU, y de las ERefU, se delimitaron unidades homogéneas de erosión para cada comuna en análisis, las que fueron clasificadas en seis niveles de susceptibilidad. En el Cuadro $N^{0} 1$ se encuentran las distintas superficies identificadas para cada comuna.

La comuna de Concón presenta mayoritariamente una vulnerabilidad a la erosión entre los rangos de ligera a moderada (2 a 4), en un $91 \%$ de su superficie (Cuadro $N^{\circ} 1$ ).

En la zona próxima a la desembocadura del río existen grados de susceptibilidad a la erosión moderada (ERU 4) en el 8\% de la superficie, mientras que en zonas de uso netamente urbano se encuentra casi el total de clases de ERU 5 y 6, correspondiendo a las más vulnerables (Figura № 3 ).

Quillota muestra principalmente una susceptibilidad a la erosión clasificada en rangos medios y ligeramente mayores que Concón, destacando la clase ERU 4 (susceptibilidad a la erosión moderada) en más del $63 \%$ de la superficie (Cuadro $\mathrm{N}^{\circ} 2$ ).

Finalmente, la comuna de Los Andes es la que presenta la mayor vulnerabilidad (Cuadro $N^{\circ} 3$ ) con el $94 \%$ de la superficie clasificada como de susceptibilidad alta y muy alta (ERU 5 y 6$)$.

Gran parte de la superficie posee suelos con una mínima cobertura vegetacional, lo cual explica la vulnerabilidad a la erosión muy alta en más del $70 \%$ del área, asociada en general a geoformas con altas pendientes, por sobre los $20^{\circ}$ (Figura $N^{\circ} 3$ ).

Cuadro $\mathrm{N}^{\circ} 1$

Superficie de Concón según Unidades de Respuesta a la Erosión (ERU)

\begin{tabular}{|c|l|c|c|}
\hline Clase de ERU & Susceptibilidad a erosión & Superficie $\left(\mathrm{km}^{2}\right)$ & Superficie $(\%)$ \\
\hline 1 & Nula & 0,7 & 1,0 \\
2 & Ligera & 31,7 & 42,3 \\
3 & Ligera - moderada & 9,0 & 12,0 \\
4 & Moderada & 27,4 & 36,5 \\
5 & Alta & 5,2 & 6,9 \\
6 & Muy alta & 1,0 & 1,3 \\
\hline
\end{tabular}

Fuente: Elaboración propia. 


\section{Aplicación de la ecuación USLE}

Se aplicó la ecuación USLE para cada una de las microcuencas seleccionadas en las tres comunas en estudio. Es posible apreciar que en general los rangos de pérdida de suelo en las tres microcuencas se encuentran entre 0-168 ton/ha año (Cuadro $\left.N^{\circ} 4\right)$. Los valores más altos se encontraron en las comunas de Concón y Los Andes, mientras que en QuiIlota son significativamente menores (Figura $\left.N^{\circ} 4\right)$.

Se aclara que dado que las microcuencas seleccionadas no son del mismo tamaño, la comparación de sus resultados es relativa.

Figura $\mathrm{N}^{\circ} 3$

Unidades de Respuesta a la Erosión (ERU) en las comunas de Los Andes, Quillota y Concón
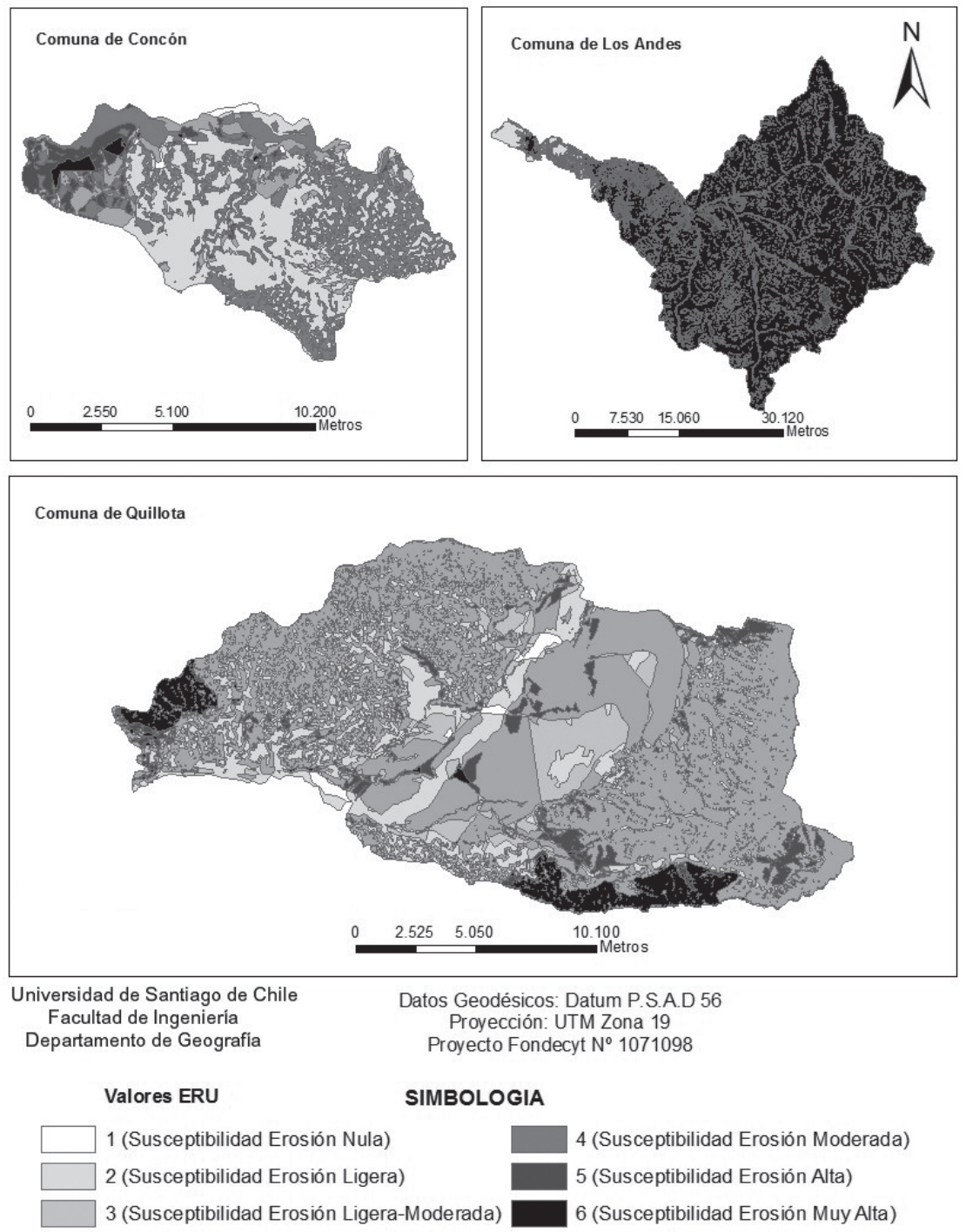

Fuente: Elaboración propia. 
Cuadro $\mathrm{N}^{\circ} 2$

Superficie de Quillota según Unidades de Respuesta a la Erosión (ERU)

\begin{tabular}{|c|l|c|c|}
\hline Clase de ERU & Susceptibilidad a erosión & Superficie $\left(\mathrm{km}^{2}\right)$ & Superficie (\%) \\
\hline 1 & Nula & 2,4 & 0,8 \\
2 & Ligera & 51,8 & 17,2 \\
3 & Ligera - moderada & 24,2 & 8,0 \\
4 & Moderada & 191,3 & 63,5 \\
5 & Alta & 15,9 & 5,3 \\
6 & Muy alta & 15,8 & 5,2 \\
\hline
\end{tabular}

Fuente: Elaboración propia.

Cuadro $\mathrm{N}^{\circ} 3$

Superficie Los Andes según Unidades de Respuesta a la Erosión (ERU)

\begin{tabular}{|c|l|c|c|}
\hline Clase de ERU & Susceptibilidad a erosión & Superficie $\left(\mathrm{km}^{2}\right)$ & Superficie (\%) \\
\hline 1 & Nula & 0,2 & 0,0 \\
2 & Ligera & 20,3 & 1,6 \\
3 & Ligera - moderada & 4,3 & 0,3 \\
4 & Moderada & 49,0 & 3,9 \\
5 & Alta & 131,5 & 10,6 \\
6 & Muy alta & $1.036,4$ & 83,5 \\
\hline
\end{tabular}

Fuente: Elaboración propia.

\section{Cuadro $\mathrm{N}^{\circ} 4$}

Pérdida de suelos en microcuencas según comunas de estudio

\begin{tabular}{|l|c|c|c|c|}
\hline Microcuenca & $\begin{array}{c}\text { Rango pérdida suelo } \\
\text { (ton/ha año) }\end{array}$ & $\begin{array}{c}\text { Superficie } \\
\left(\mathrm{km}^{2}\right)\end{array}$ & $\begin{array}{c}\text { Superficie } \\
\text { (ha) }\end{array}$ & $\begin{array}{c}\text { Pérdida suelo } \\
\text { (ton/año) }\end{array}$ \\
\hline Concón & $0-119,7$ & 1,8 & 184,3 & $5.060,6$ \\
Quillota & $0-60,2$ & 29,4 & $2.942,1$ & $46.602,3$ \\
Los Andes & $0-168,2$ & 8,1 & 809,4 & $24.271,5$ \\
\hline
\end{tabular}

Fuente: Elaboración propia. 
Así, las pérdidas totales (ton/año) resultan proporcionales a su superficie, por tanto, Quillota, con la microcuenca más grande $\left(29,4 \mathrm{~km}^{2}\right)$, presenta las pérdidas totales mayores, aunque cuenta con un rango menor de pérdida por hectárea.

\section{Consideraciones finales}

A través de la aplicación conjunta de los métodos de la USLE y ERU fue posible evaluar la susceptibilidad a la erosión en términos cualitativos y cuantitativos, en distintos

Figura $\mathrm{N}^{\circ} 4$

Pérdida de suelo según USLE, aplicada a las comunas de Los Andes, Quillota y Concón
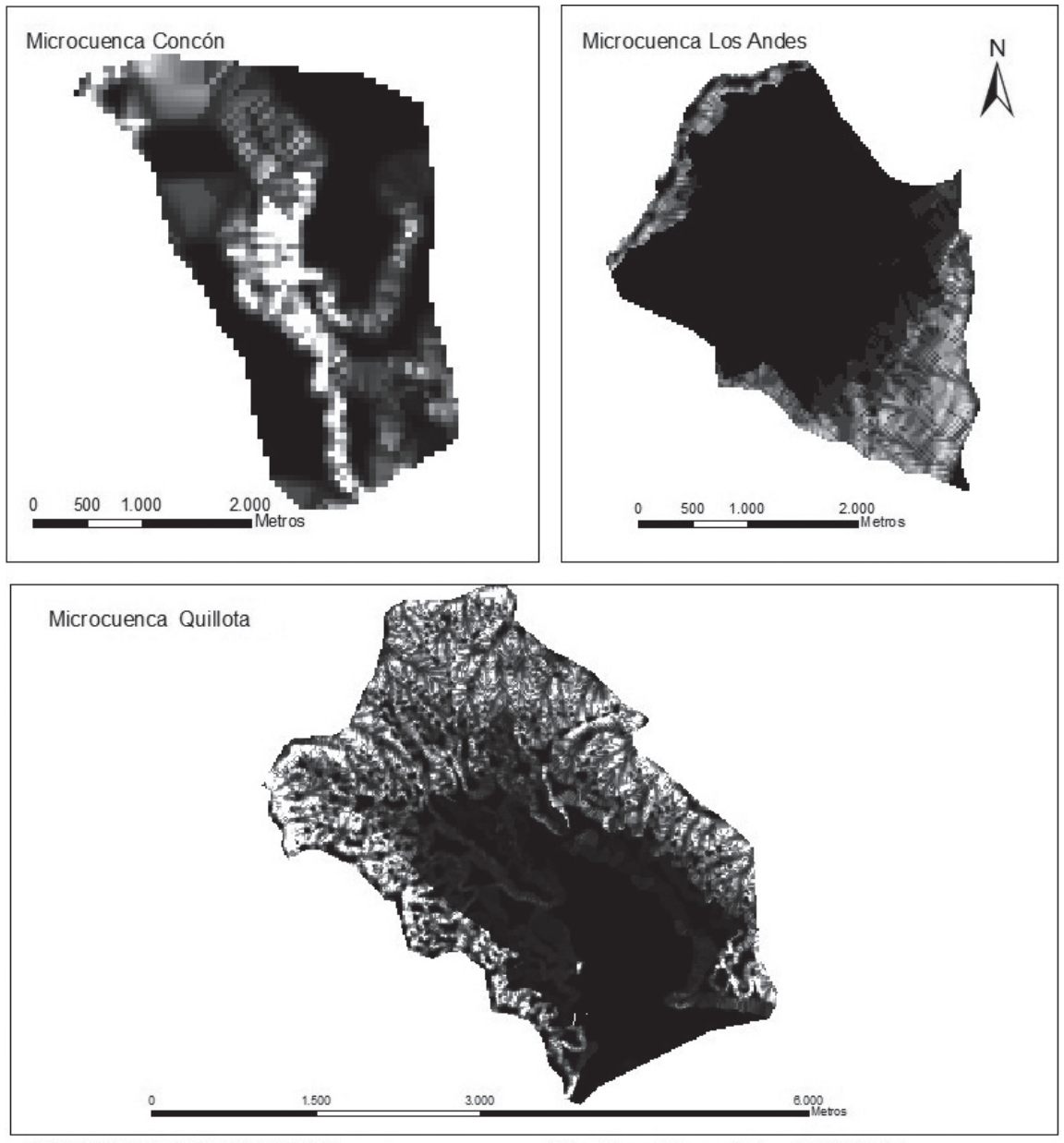

Universidad de Santiago de Chile

Facultad de Ingeniería

Departamento de Geografia

Pérdida Suelo (ton/ha.año)

SIMBOLOGÍA

Datos Geodésicos: Datum P.S. A.D 56

Proyección: UTM Zona 19

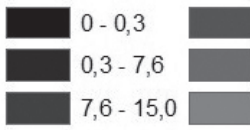

$15,0-25,5$

$46,4-61,6$ 109

$109,5-170$

$25,5-39,0$

$61,6-78,3$

$39,0-46,4$

$78,3-109,5$

Fuente: Elaboración propia. 
ambientes del valle del Aconcagua, lo que permite realizar un diagnóstico preliminar de la erosión actual natural y antrópica y de la vulnerabilidad ante ella en la zona.

El factor que resultó claramente condicionante de la susceptibilidad a la erosión, además de la pendiente, es el uso de suelo en conjunto con la cobertura vegetal natural actual. La erosión alta y muy alta se encuentra asociada a superficies desprovistas de vegetación, donde el suelo se encuentra sin protección frente a la acción erosiva de las precipitaciones. Susceptibilidades de erosión ligera a moderada están asociadas a usos de bosques, suelos agrícolas, matorrales y praderas. Este factor está íntimamente ligado a la componente topográfica, donde suelos de altas pendientes, altamente vulnerables a la degradación, sin cobertura vegetacional, presentan mayores posibilidades de erosionarse.

Actualmente, las prácticas agrícolas del valle del Aconcagua favorecen ambas condicionantes, donde, especialmente en la etapa de preparación de terrenos para el cultivo, la eliminación casi total de la cubierta vegetacional favorece una significativa pérdida de suelos. La erosividad pluvial no resultó ser un factor que genere grandes diferencias en los resultados obtenidos para las Unidades de Respuesta a Erosión (ERU) entre los distintos sectores del valle, ya que se trata en general de altos valores erosivos en las distintas secciones de la cuenca que fueron analizadas.

En contraste con esto, la diferenciación de las Unidades Homogéneas de Respuesta a la Erosión (ERU), por su parte, se vio influenciada en gran medida por las diferencias texturales del suelo. Así, erosiones ligeras se asocian a texturas moderadamente finas, con alto porcentaje de arcilla, que en conjunto con la materia orgánica aporta estabilidad a los suelos y, a su vez, regulan la infiltración de agua evitando saturaciones tempranas del suelo frente a eventos de precipitación significativos.

En cuanto a una diferenciación de los resultados entre las comunas según las ERU, la cantidad de superficie susceptible a ser erosionada decrece según su posición altitudinal en el valle del Aconcagua. De esta manera, la comuna de Los Andes es más vulnerable debido a sus suelos desnudos, pendientes pronunciadas y falta de manejo apropiado del suelo. No sucede lo mismo en las comunas de Quillota y Concón, que se encuentran en una posición intermedia y costera, respectivamente, con pendientes moderadas y aplicación de técnicas de manejo de cultivos.

Por otro lado, los resultados de la aplicación de la USLE en las microcuencas definidas para las tres comunas, son congruentes con aquellos entregados por la modelación de las zonas homogéneas de vulnerabilidad erosiva, modeladas a escala regional. Al comparar las pérdidas de suelo por superficie de cada microcuenca, los resultados muestran que existen pérdidas altas en la microcuenca de la comuna de Los Andes (30,0 ton/ año/ha). Luego, la microcuenca de Concón presenta valores medios-altos de pérdida de suelo en su superficie $(27,5$ ton/año/ha). Finalmente, en la microcuenca de Quillota se pierden cerca de 15,8 ton/año/ha.

\section{Referencias bibliográficas}

ALIAGA, C. y LEYTON, N. Evaluación de la pérdida de suelo y de su calidad, asociada al proceso de expansión urbana y reconversión productiva, Valle del Aconcagua, comunas de Los Andes, Quillota y Concón. Trabajo título profesional de Ingeniería Civil en Geografía. Santiago: Departamento de Ingeniería Geográfica, Facultad de Ingeniería, Universidad de Santiago de Chile, Chile, 2007.

CASTRO, C. P. y ORTIZ, J. Expansión urbana y niveles de vulnerabilidad a amenazas naturales en una ciudad tamaño medio: La Serena, Región IV de Coquimbo. En: HIDALGO, R.; TUMPER, R. y BORSDORF, A. (eds.). Transformaciones urbanas y procesos territoriales del nuevo dibujo de la ciudad latinoamericana. Santiago: Pontificia Universidad Católica de Chile, Instituto de Geografía, Serie GEOlibros 4, 2005, p. 299-306.

FOURNIER, F. Conservación de suelos. Madrid: Ediciones MUNDI-PRENSA, 1975.

HONORATO, R.; BARRALES, L.; PEÑA, I. y BARRERA, F. Evaluación del modelo USLE en la estimación de la erosión en seis localidades entre la IV y IX región de Chile. Revista Ciencias de la Agricultura, 2001, vol. 28, $\mathrm{N}^{\circ}$ 1, p. 7-14. 
HONORATO, R. y CRUZ, G. Erodabilidad y erosión actual de los suelos de Isla de Pascua. Revista Ciencia e Investigación Agraria, 1999, № 26, p. 27-36.

MÄRKER, M.; MORETTI, S. \& RODOLFI, G. Assessment of water erosion processes and dynamics in semi-arid regions of southern Africa (kwazulu/Natal, RSA, and Swaziland) using the Erosion Response Units concept
(ERU). Revista Geogr. Fis. Dinam. Quat., 2001, vol. 24, issue 1, p. 71-84.

VAN ZUIDAM, R. Landscapes in the tropical humid and subhumid climatic zones. In: INTERNATIONAL INSTITUTE FOR AEROSPACE SURVEY AND EARTH SCIENCES. Aerial photo-interpretation in terrain analysis and geomorphological mapping. The Netherlands: ITC, 1985. 